\title{
Engineer complete resistance to Cotton Leaf Curl Multan virus by the CRISPR/Cas9 system in Nicotiana benthamiana
}

\author{
Kangquan Yin ${ }^{1,2 \dagger}$, Ting Han ${ }^{1 \dagger}$, Ke Xie ${ }^{3 \dagger}$, Jinping Zhao ${ }^{1,4,5}$, Junqi Song ${ }^{4,5}$ and Yule Liu ${ }^{1 *}$
}

\begin{abstract}
Clustered, regularly interspaced short palindromic repeats/CRISPR-associated proteins (CRISPR/Cas) based genome editing technology has been developed from the adaptive immune system used by many bacteria and archaea for combating against viruses. Here, we utilize the Cas9 with dual gRNAs designed to target two essential regions of the single-stranded DNA genome of the Cotton Leaf Curl Multan virus (CLCuMuV) which can cleavage the corresponding DNA sequence and thus interrupt viral infection. The transgenic plants expressing Cas9 and dual gRNAs that target different regions of the CLCuMuV genome confer complete resistance to virus infection, thereby demonstrating a novel approach for engineering resistance to geminiviruses.
\end{abstract}

Keywords: Genome editing, Virus resistance, Geminivirus

\section{Background}

There are several strategies to engineer resistance against viral diseases in plants, the most powerful of which is based on pathogen-derived resistance (PDR), a concept referring to the insertion of viral sequences in plant cells leading to virus resistance (Beachy 1997). PDR can be grouped into two classes: protein-mediated resistance and RNAi-mediated resistance (Beachy 1999; Baulcombe 2004) and has been widely and successfully applied in the anti-RNA virus field. Many commercial transgenic plants have been engineered in this way, including SunUp, a genetically modified papaya variety with transgenic viral coat protein that is resistant to ringspot virus (Ming et al. 2008). However, PDR-based DNA virus resistance has not been exploited as successful as that against RNA viruses, as DNA and RNA viruses use distinct pathogenic mechanisms to infect host plants. Therefore, a new approach needs to be developed to combat DNA viruses.

\footnotetext{
* Correspondence: yuleliu@mail.tsinghua.edu.cn

† Kangquan Yin, Ting Han and Ke Xie contributed equally to this work.

${ }^{1}$ Center for Plant Biology, MOE Key Laboratory of Bioinformatics, School of

Life Sciences, Tsinghua University, Beijing 100084, China

Full list of author information is available at the end of the article
}

CRISPR/Cas9-based genome editing technology was recently developed from a defense mechanism used by bacteria and archaea for control of viruses. This system is composed of two components: a programmable DNA nuclease Cas9 and a short RNA called guide RNA (gRNA). The Cas9 nuclease forms a complex with a gRNA and searches for the DNA target that is complementary to the first $20 \mathrm{nt}$ of the gRNA, which has a 5'-NGG-3' PAM (protospace adjacent motif) immediately downstream of the complementary region. When the complex finds the target, the Cas9 will cut both strands of the target DNA at around $3 \mathrm{bp}$ upstream of the PAM. Shortly after this technology was first demonstrated to serve a function in human cells, it was extended and applied to many other species including mouse, worm, fly, fish and plant (Hsu et al. 2014).

Cotton is one of the most important economic crops across the globe. However, in the past two decades, cotton production in Asian countries such as Pakistan, India and China, was threatened by the epidemic of cotton leaf curl disease (CLCuD) (Briddon and Markham 2000). CLCuD is transmitted exclusively by whitefly Bemisia tabaci, which carries the $\mathrm{CLCuD}$ pathogen Begomoviruses, causing leaf curling, vein darkening, vein swelling, and enations on the underside of leaves, among other characteristic symptoms (Briddon and Markham 2000). Begomoviruses belonging to

(c) The Author(s). 2019 Open Access This article is distributed under the terms of the Creative Commons Attribution 4.0 International License (http://creativecommons.org/licenses/by/4.0/), which permits unrestricted use, distribution, and reproduction in any medium, provided you give appropriate credit to the original author(s) and the source, provide a link to the Creative Commons license, and indicate if changes were made. The Creative Commons Public Domain Dedication waiver (http://creativecommons.org/publicdomain/zero/1.0/) applies to the data made available in this article, unless otherwise stated. 
the family Geminiviridae are single-stranded DNA (ssDNA) viruses and are often associated with single-stranded DNA satellite (DNA $\beta$ ). Although Begomoviruses are isolated from cotton, they can infect Nicotiana benthamiana plants and cause characteristic symptoms. Among the various members of Begomovirus species, Cotton Leaf Curl Multan virus $(\mathrm{CLCuMuV})$ is the most devastating virus responsible for huge cotton production losses in the northern-western India subcontinent since the 1980s. The virus has also spread to China (Cai et al. 2010).

$\mathrm{CLCuMuV}$ is a monopartite Begomovirus typically associated with a single-stranded satellite molecule called CLCuMuB. The $2.7 \mathrm{~kb}$ CLCuMuV genomic DNA consists of six protein coding genes designated as $\mathrm{V} 1, \mathrm{~V} 2, \mathrm{C} 1, \mathrm{C} 2, \mathrm{C} 3$, and $\mathrm{C} 4$, which encode capsid protein $(\mathrm{CP})$, precoat protein, replication-associated protein (Rep), transactivator protein (TrAP), replication enhancer protein (REn), and C4 proteins, respectively (Fig. 1a) (Cai et al. 2010). When CLCuMuV enters the host plant cell via its insect vector whitefly, it uncoats and releases its ssDNA genome into the nucleus. The ssDNA genome is then converted into a covalently closed double-stranded DNA (dsDNA) circle as the replicative form (RF). DNA motifs in the intergenic region (IR) of the RF are used by host polymerase to generate viral mRNAs through bidirectional transcription. The viral mRNAs are subsequently translated into viral proteins. Once the Rep protein is made, it nicks the plus strand of the invariant sequence in the IR to initiate the replication of viral genome via rolling-circle replication (RCR) mechanism. In the early stage of RCR process, double-stranded RF DNAs are generated while in the later stage of RCR, the ssDNA genomes accumulate. The ssDNA viral genomes are finally packaged into the $\mathrm{CP}$ and released into neighboring plant cells through plasmodesmata (Fig. 1b).

\section{Results \\ sgRNA design}

It is hypothesized that if the RF DNA of CLCuMuV can be targeted directly by the CRISPR/Cas9 system, $\mathrm{CLCuMuV}$ should be suppressed or cleared by the consequently mutagenizing key genomic elements or destabilizing the viral replication intermediates through linearization of circular genomes. To inhibit the replication of $\mathrm{CLCuMuV}$, we in silico designed all the gRNAs targeting the IR region and the $\mathrm{C} 1$ coding region via the gRNA Finder tool (http://crispr.hzau.edu.cn/cgi-bin/CRISPR2/SCORE) (Additional file 1: Table S1). Following the gRNA design principle by Doench et al. (2016) we selected one gRNA with potential high activity each for the IR region (gRNA-1) and the $\mathrm{C} 1$ coding region (gRNA-2), respectively.

\section{Test of sgRNA activity}

To test the gRNAs' activity in plant cells, we adopted an $f_{s} G F P$ reporter system similar to our previous $f_{s} G U S$ reporter system (Fig. 2a) (Yin et al. 2015), in which the gRNA targeting sequence was inserted right after the start codon leading to disruption of the reading frame of GFP. Once the Cas9 and gRNA co-expressed in the cell, the fsGFP DNA was targeted and cut by the Cas9. The resulting double strand break (DSB) were repaired by non-homologous end joining (NHEJ) and $f_{S} G F P$ could possibly be restored to a functional GFP gene. To test the activity of gRNA-1 in the plant, $N$. benthamiana plant leaves were co-infiltrated with two Agrobacterium strains with one harboring a binary vector expressing the Cas 9 gene by $35 \mathrm{~S}$ promoter and gRNA-1 by U6 promoter and the other harboring the $f_{S} G F P$ gene (Fig. 2a). In $~ 50 \%$ leaves examined at 48 hours after infiltration, a number of cells displayed strong fluorescence under confocal microscopy. However, no fluorescence was observed when leaves were infiltrated with Agrobacterium containing either Cas 9 or $f S G F P$ alone. The same assay was applied to gRNA-2 and only leaves infiltrated with Agrobacterium containing Cas $9, g R N A-2$ and $f s G F P$ displayed strong fluorescence under confocal microscopy (Fig. 3a).

We confirmed the above results and investigated the mutations caused by the Cas9/gRNA system at the target site of the $f_{s} G F P$ gene in leaf cells converting the non-functional $f_{s} G F P$ gene into a functional GFP gene. In our investigation, we extracted DNA from the leaf regions with fluorescence. The DNA samples underwent PCR amplification using primers flanking the targeting site of each gRNA in $f_{s} G F P$ gene. PCR amplicons were then cloned into vectors and sequenced. The sequencing results showed that both gRNA-1 and gRNA-2 are active gRNAs, which can guide Cas9 protein to cut the target sites in the $f_{S} G F P$ gene, generating variable mutations through NHEJ (Fig. 3b). Most mutations are +1 and -1 frameshifts, consistent with previous reports (Shan et al. 2013). Moreover, some mutations generated in-frame coding sequence of GFP gene, thus reconstituting a functional GFP (Fig. 3b). Taken together, these results showed both gRNAs are highly active for gene editing.

\section{Transgenic plants with Cas9-gRNA confer virus resistance}

The successful cleavage of $f_{s} G F P$ genes mediated by Cas9 and the two designed gRNAs provided the possibility of using these two gRNAs to mediate the cleavage of geminivirus dsDNA intermediates with an aim to increase resistance to virus attack. To this end, one construct (p35S:oCas9-U6:gRNA-1-U6:gRNA-2) with expression of Cas9 and the two gRNAs simultaneously was transformed into $N$. bentahmiana plants by Agrobacterium-mediated transformation (Fig. 2b). The transgenic plants carrying Cas 9 and gRNAs were identified. We selected two 
a
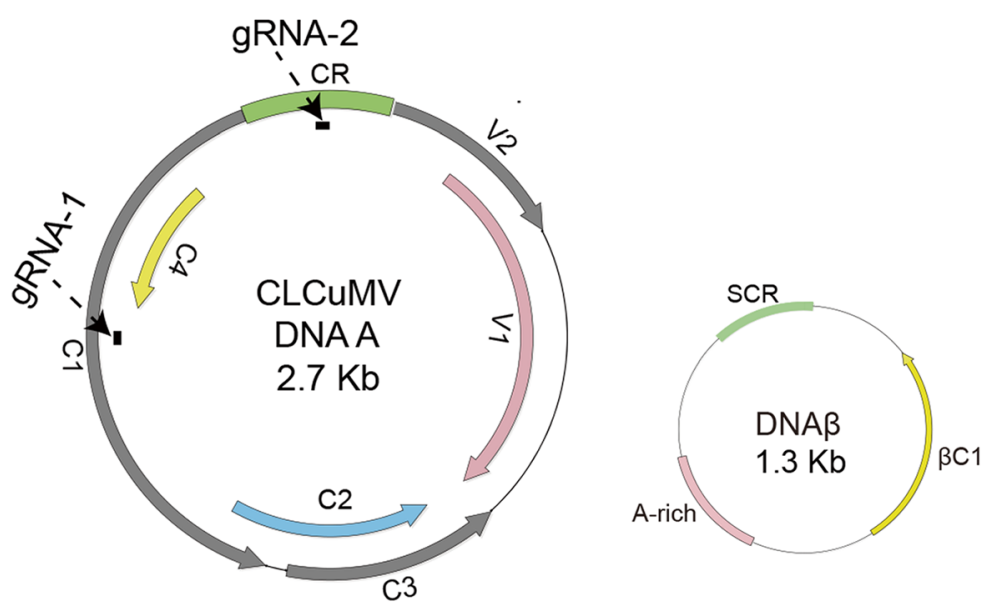

b

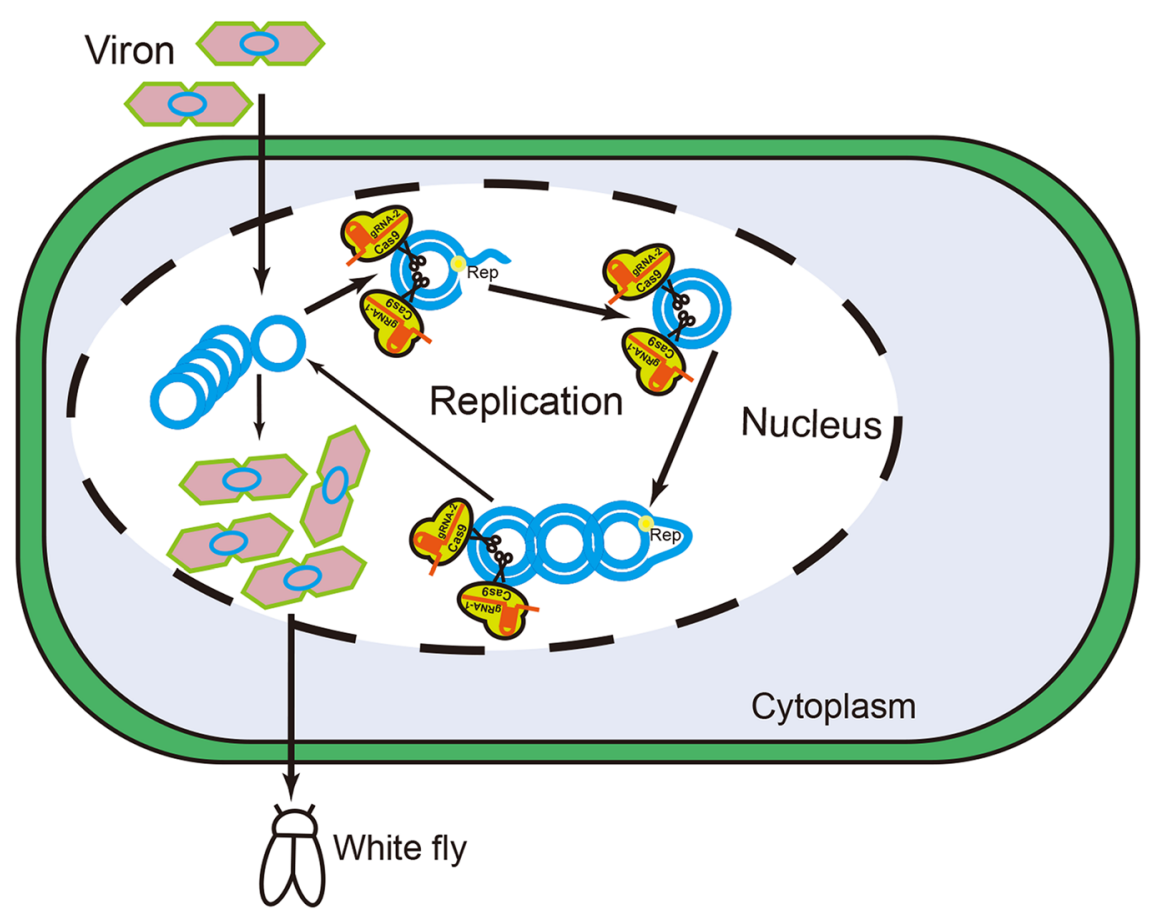

Fig. 1 Schematic diagrams of constructions of CLCUMuV and CLCUMuB infectious clones and CRISPR/Cas9 system-mediated virus resistance in plants. a Genome organization of the wild-type CLCuMuV and CLCUMuB and sgRNA target sites. The ORFs are represented by filled arrows, CR, SCR and A-Rich region are represented by filled boxes. The two target sites are represented by dashed arrowheads. $\mathbf{b}$ Schematic representation of CRISPR/Cas9 system-mediated CLCuMuV resistance. Once the virus infects a plant cell, it enters the cell nucleus where it replicates to produce more viruses. Some of them are packaged into virus, which can be transmitted into other plants by whitefly

transgenic lines (nos. 1 and 8) and challenged their progeny plants with CLCuMuV. Wild-type plants and Cas9-only transgenic plants were used as controls.

Thirty-five days post inoculation (dpi), all the wild-type and Cas9-only transgenic plants showed typical CLCuMuV-induced symptoms such as severely downward leaf curling, darkening, shorter stem internodes and abnormal flowers (Fig. 4). By contrast, transgenic plants expressing Cas9, gRNA-1 and gRNA-2 appeared to grow normally, resembling mock-inoculated wild-type and Cas9-only transgenic plants (Fig. 4).

The total DNA from plants in Fig. 4 were extracted and assayed by Southern blot using a biotin-labeled hybridization probe specific to CLCuMuV. A typical circular DNA pattern as well as ss- and dsDNA profiles of $\mathrm{CLCuMuV}$ were readily detected in high abundance in both wild-type and Cas9-only transgenic plants (Fig. 5). By contrast, CLCuMuV was undetectable in transgenic 


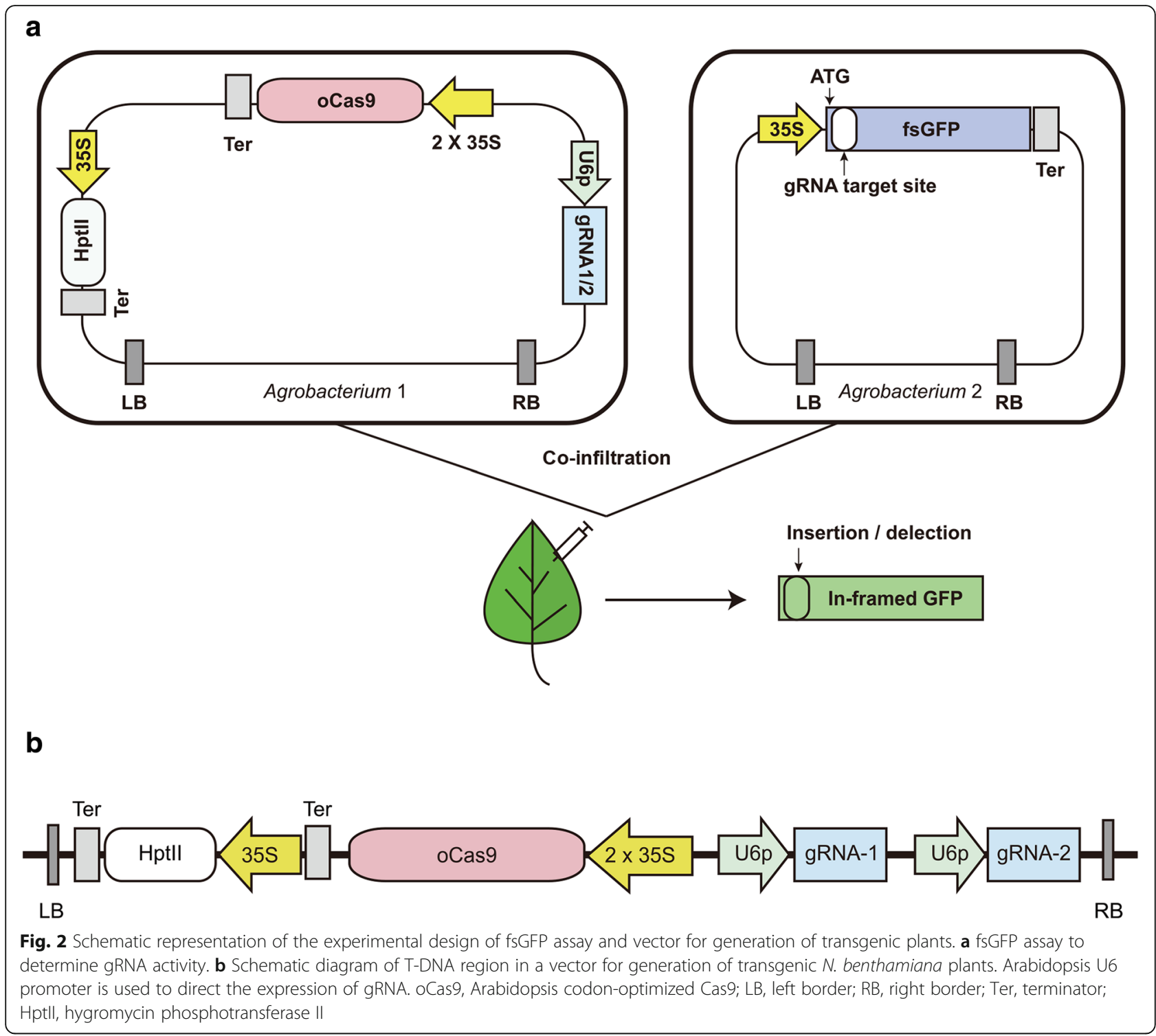

plants simultaneously expressing Cas 9 , gRNA-1 and gRNA-2, indicating that high virus resistance was achieved in these lines (Fig. 5). Overall, our results show that transgenic plants generated by the CRISPR/Cas9 system in combination with the dual gRNAs, confer complete $\mathrm{CLCuMuV}$ resistance.

\section{Discussion}

Geminiviruses are characterized by their twinned icosahedral particles. They can infect a lot of plants including staple food crops, fiber crops, weeds and ornamental plants, causing great yield losses around the world (Hanley-Bowdoin et al. 2013). Although PDR is the most powerful approach to generate resistance to RNA viruses, it was not successful to geminiviruses. For example, transgenic expression of C-terminal truncated forms (Rep210) of the Rep of Tomato yellow leaf curl
Sardinia virus (TYLCSV) confers resistance to this virus by inhibiting its transcription and replication. However, this resistance to TYLCSV is not durable because virus could quickly silence the Rep-210 transgene via virus-induced gene silencing (VIGS) (Lucioli et al. 2003). In addition, transgenic expression of Bean golden mosaic virus (BGMV) coat protein gene failed to confer resistance to this virus (Russell et al. 1993). While transgenic expression of replication initiator protein gene, transactivator protein, replication enhancer protein gene and movement protein gene in an antisense orientation could delay golden mosaic symptoms after inoculation via whitefly (Aragão et al. 1998; Aragão and Faria 2009). Furthermore, transgenic expression of the mutated Rep gene conferred resistance to BGMV but that resistance is dependent on the dosage of virus inoculated (Faria et al. 2006). Therefore, alternative approaches other than 


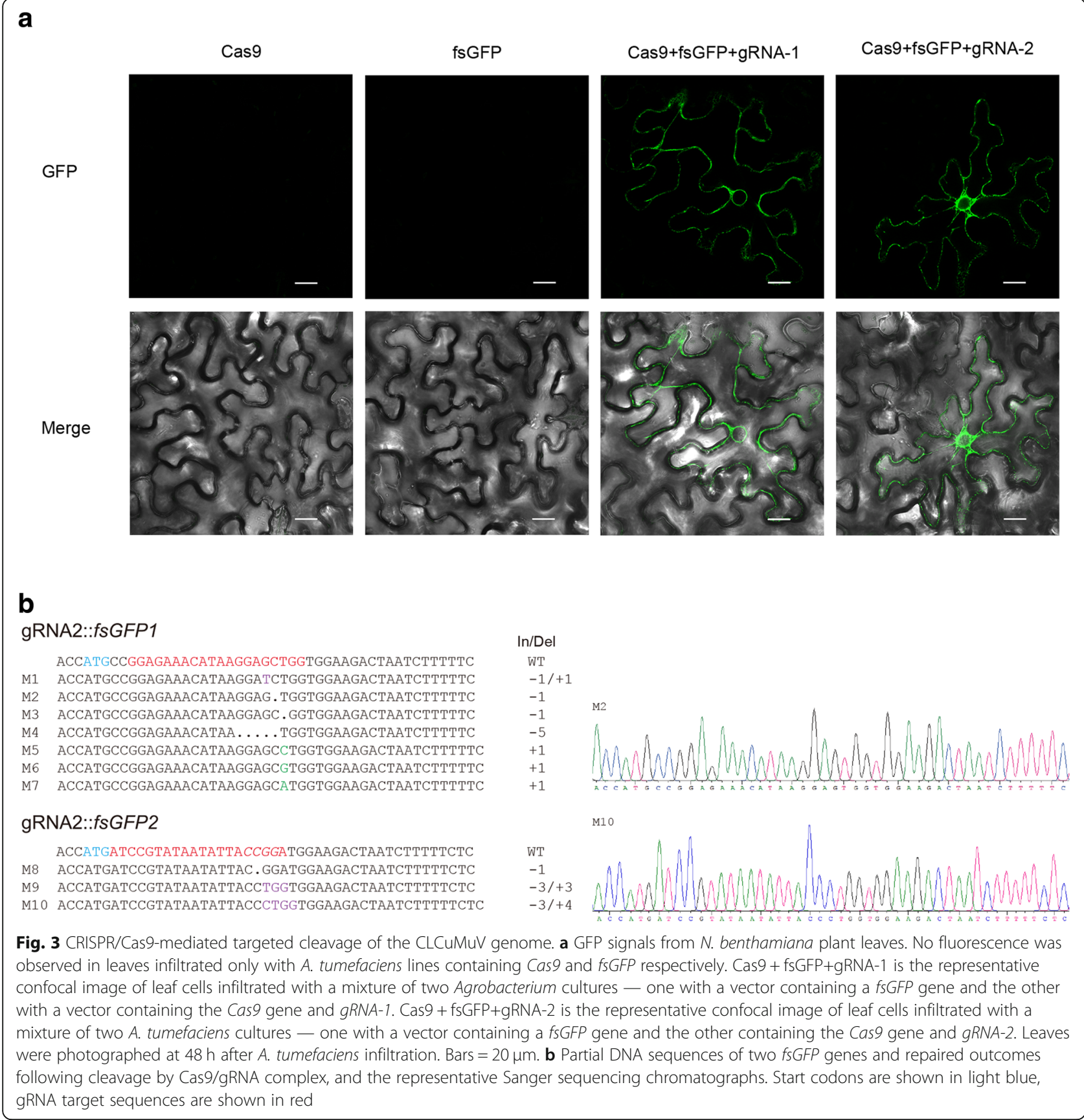

PDR are urgently required for preventing geminivirus infections in crop plants.

The development of genome editing systems provides potential controls against DNA viruses. The first artificial zinc finger protein (AZP) targeting a $19 \mathrm{bp}$ fragment in the IR region of Beet severe curly top virus (BSCTV) in planta was designed in the mid-2000s (Sera 2005). Because the IR region contains a binding site recognized by the Rep protein, BSCTV replication was reduced in AZP transgenic plants in which AZP would block the Rep binding site (Sera 2005). Unlike AZP, zinc finger nuclease (ZFN) mediated DNA virus resistance involves not only the binding of virus, but also the cleavage of virus and the introduction of deleterious mutations in the viral genome. ZFN technology was recently used to provide resistance against Tomato yellow leaf curl China virus (TYLCCNV) by targeting the Rep gene (Chen et al. 2014). However, its efficient application in transgenic plants remains to be confirmed. Transcription activator like effectors (TALEs) were recently developed to target two conserved $12 \mathrm{nt}$ sequences among begomoviruses and these TALEs provided broad-spectrum resistance 


\section{a}

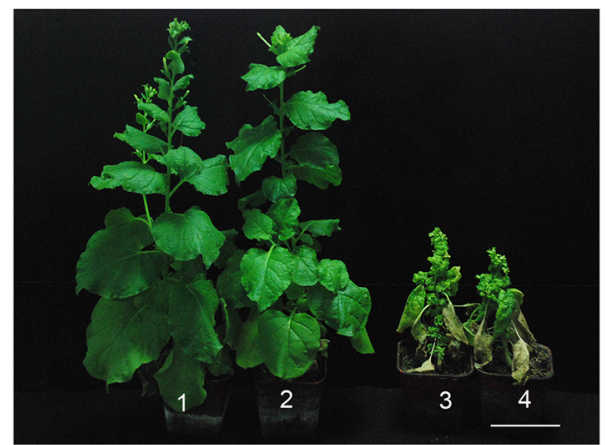

1.Cas9+gRNA-1+gRNA-2+CLCuMV_line1

2.Cas9+gRNA-1+gRNA-2+CLCuMV_line8

3. Cas9+CLCuMV

4.nn+CLCUMV b

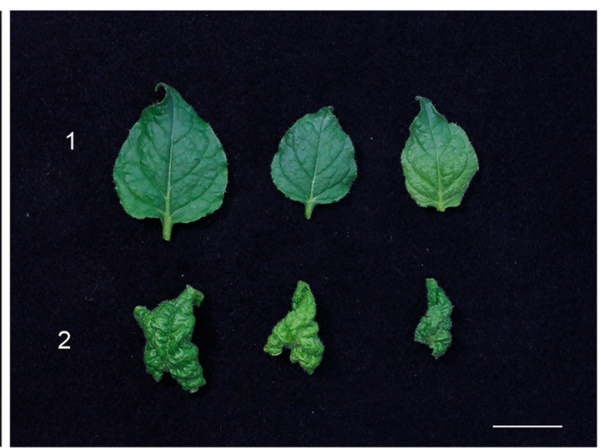

1.Cas9+gRNA-1+gRNA-2+CLCuMV

2.Cas9+CLCUMV

Fig. 4 Symptoms on N. benthamiana plants inoculated with the infectious CLCuMuV and CLCuMuB clones. a Representative images of control and transgenic plants 35 days (dpi) with CLCuMuV and CLCuMuB. 1 to 4 represent Cas9 + gRNA-1 + gRNA-2 transgenic line1, Cas9 + gRNA-1 + gRNA-2 transgenic line8, Cas9 transgenic, and WT, respectively. Bar $=8 \mathrm{~cm}$. $\mathbf{b}$ The phenotype of the third, fourth and fifth leaves at the top of transgenic plants 20 dpi with CLCuMuV and CLCuMuB. Group 1 and 2 represent Cas9 + gRNA-1 + gRNA-2 transgenic line1 and Cas9 transgenic, respectively. Bar $=3 \mathrm{~cm}$

against three DNA viruses, including Tobacco curly shoot virus (TbCSV), Tomato leaf curl Yunnan virus (TLCYnV) and TYLCCNV (Cheng et al. 2015). However, TALE nucleases (TALENs) have not been reported to provide resistance against DNA viruses, partly due to the difficulty and cost. In contrast, the simplicity, efficiency and affordability of CRISPR-Cas9 technology attracted a lot of attentions immediately after its first application in eukaryotic cells. Three studies have shown that the CRISPR/Cas9 system can be used to engineer resistance to DNA viruses (Ali et al. 2015; Baltes et al. 2015; Ji et al. 2015). They all employed a single gRNA to target corresponding DNA viruses. In this study, we used two gRNAs to target IR and $\mathrm{C} 1$ responsible for the rapid replication of CLCuMuV, and generated CRISPR-edited plants that confer complete resistance to $\mathrm{CLCuMuV}$. The ability of DNA viruses to evade the CRISPR/Cas9 cleavage has been recently evaluated. They found that although CRISPR/Cas9 can efficiently target the coding region of different DNA viruses with one sgRNA, the NHEJ mediated repair also resulted in the generation of viral variants with replication and movement abilities (Ali et al. 2016). However, CRISPR/Cas9 can simultaneously target the coding region with two nearby sgRNAs and generate relatively large indels which will disrupt the coding region of a DNA virus. Even if the two sgRNAs did not work simultaneously and thus no indels will occur, NHEJ mediated repair of two nearby sites will have extremely low chance to generate viral variants with functional protein corresponding to the target gene. Therefore, two or more
sgRNAs will increase the CRISPR/Cas9 mediated immunity to DNA viruses.

Field plants are threatened by single virus species and many other DNA viruses. Thus, it is essential to control viral diseases in a simple and efficient manner. There are three alternative ways to engineer resistance to DNA viruses. The first way is to efficiently target the most conserved region of a group of DNA viruses or even all the DNA viruses. For example, Cabbage leaf curl virus and African cassava mosaic virus have nearly identical 200$250 \mathrm{bp}$ common regions (Hanley-Bowdoin et al. 2000). Designed sgRNAs from these regions will target both viruses and have potential to generate broader resistance than sgRNAs from other non-conservative regions. Interestingly the IR regions of all the geminiviruses contain an invariant sequence: TAATATTAC. However, this 9 bp sequence is too short and cannot be targeted by current CRISPR/Cas systems. In the future, modified versions of CRISPR/Cas system based on structure knowledges may recognize this short sequence and do the cleavage in all the geminiviruses. The second way is to express multiple sgRNAs targeting as many DNA viruses as possible in a transgenic plant. This strategy takes advantage of the multiplicity of the CRISPR/Cas systems. There are several methods reported to efficiently produce multiple sgRNAs, including tRNAgRNA systems, Csy4 endoribonuclease mediated sgRNA production and Ribozyme-gRNA-Ribozyme (RGR) system (Gao and Zhao 2014; Nissim et al. 2014; Xie et al. 2015). However, all these methods can produce no more than 10 sgRNAs, which is far less than the number of 


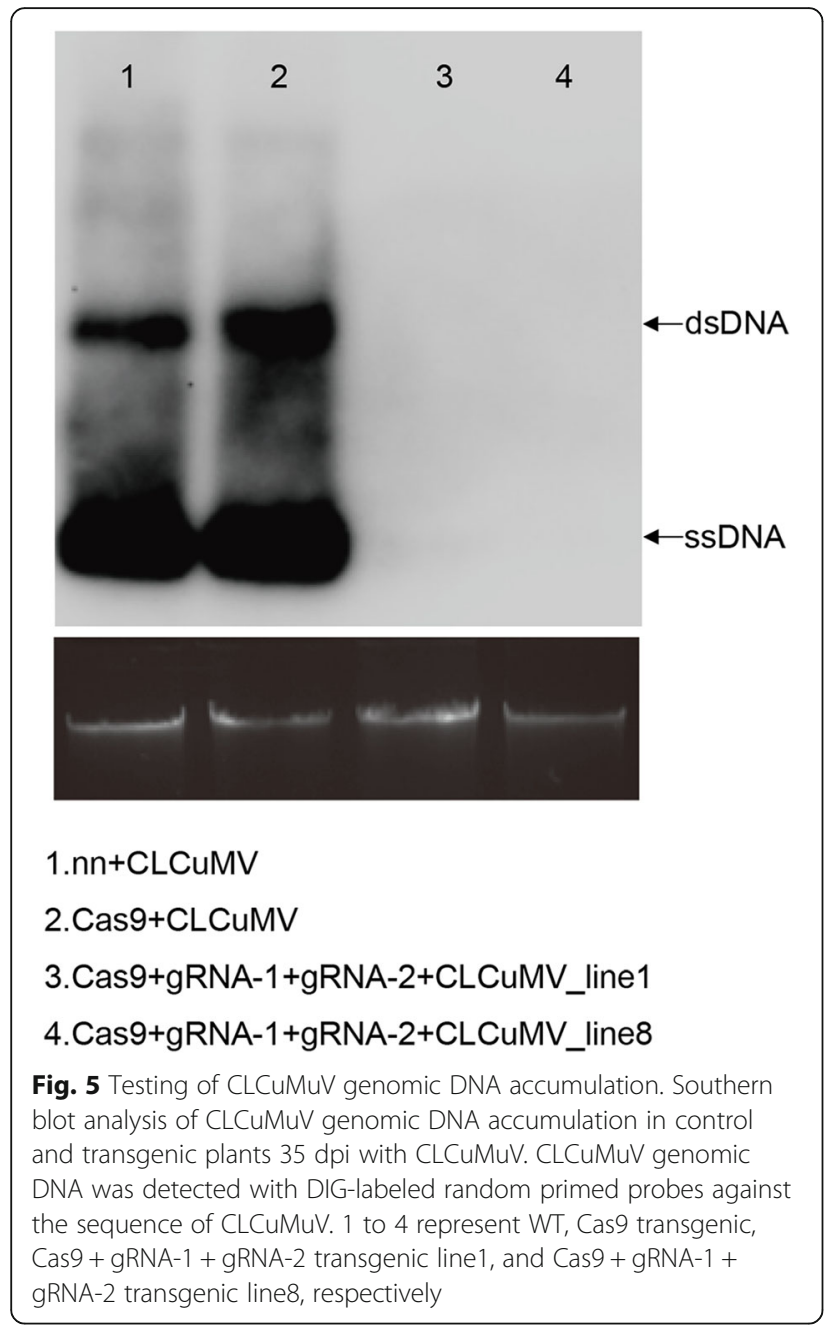

DNA viruses. Therefore, new methods that could produce a lot more sgRNAs are needed. The third way is to modify host factors like the transmembrane immune receptor NIK1 to generate broader spectrum resistance to geminiviruses (Brustolini et al. 2015; Zorzatto et al. 2015).

In nature, geminiviruses are transmitted by insect vectors. For example, white flies (Bemisia tabaci) acquire virions of begomoviruses during their feeding on the infected plants. The virions are then transmitted to another plant during the next feeding cycle of the white flies. Currently, all the reported resistances to geminiviruses via CRISPR/Cas systems were based on the challenge results of agroinoculations. Although this method resembled natural inoculation, the differences of virion load between these two inoculation methods have not been systematically evaluated. In the future, to ensure that engineered resistances to geminiviruses based on CRISPR/Cas systems are efficient in the field, it is better to perform inoculation on cotton and other test plants with white flies.

\section{Conclusions}

Our results demonstrate that, through expressing Cas9 and dual gRNAs to simultaneously target multiple regions of DNA viruses, we can engineer plants with remarkably complete resistance to DNA viruses for effective viral disease control.

\section{Methods}

\section{Plasmid construction}

Two gRNAs targeting CLCuMuV (gRNA-1 and gRNA-2) were firstly cloned into the pT-U6p-scaffold-U6t vector (Yin et al. 2015) respectively by inverse PCR mutagenesis method to generate pT-U6p-gRNA1-scaffold-U6t and pT-U6p-gRNA2-scaffold-U6t. To generate a vector that can accommodate both Cas9 expression cassette and gRNA expression cassette, pKQ334 vector (Yin et al. 2015) which contains the Cas9 expression cassette was modified. The fragment LIC5-ccdB-Chl-LIC6 containing $c c d B$ gene and chloramphenicol resistance gene was amplified by oYK782 and oYK783 using pJG081 as template and inserted into PmeI site of pKQ334 by Gibson assembly. The resulting vector was named pKQ334-LIC. gRNA expression cassette was amplified using pT-U6p-gRNA1-scaffold-U6t or pT-U6p-gRNA2scaffold-U6t as template with primers oYK792 and oYK793. These two PCR products were then inserted into pKQ334-LIC by ligation independent cloning method to generate pKQ334-gRNA-1 and pKQ334-gRNA-2, respectively.

To construct fsGFP (frame shift GFP) for testing gRNA1 activity, the gRNA-1 target sequence was first inserted after start codon of a modified mGFP5 (Ruiz et al. 1998) using primer oYK1530 and oYK1532. The PCR product was then cloned into pjg081 by LIC method to generate pjg081-fsGFP-CLCuMV-gRNA1. In this vector, the fs GFP gene was driven by a duplicate $35 \mathrm{~S}$ promoter. For testing gRNA-2 activity, pig081-fsGFP-CLCuMV-gRNA2 vector was constructed the same way as that for gRNA-1 using primer oYK1531 and oYK1532.

gRNA-1 expression cassette was first PCR amplified with primers oYK1264 and oYK1265 using pT-U6p-gRNA1-scaffold-U6t as template. The PCR product was then cloned into the $K p n \mathrm{I}$ and $\mathrm{XbaI}$ site of pT-U6p-gRNA2-scaffold-U6t to generate pT-U6pgRNA1-scaffold-U6t-U6p-gRNA2-scaffold-U6t. A $\sim 2$ kb DNA fragment containing gRNA-1 and gRNA-2 expression cassettes was PCR amplified with primers oYK1291 and oYK1292 using pT-U6p-gRNA1-scaffold-U6t-U6p-gRNA2-scaffold-U6t as template. The PCR product was cloned into the pKQ334-LIC using 
LIC method and the resulting binary vector was p35S:oCas 9-U6:gRNA-1-U6:gRNA-2.

\section{Generation of transgenic plants}

Transgenic plants were generated with Agrobacterium-mediated leaf disc method (Horsch et al. 1985; Yin et al. 2015). Briefly, leaves of 4-8 weeks old wild type $N$. benthamiana plants (nn) were first harvested and surface sterilized with $20 \%(\mathrm{v} / \mathrm{v}) \mathrm{NaClO}$ plus $0.1 \%(\mathrm{v} / \mathrm{v})$ Tween 20 for $10 \mathrm{~min}$. Then the leaves were rinsed using sterilized water with $0.1 \%(\mathrm{v} / \mathrm{v})$ Tween 20 for 4 times. 1 $\mathrm{cm}$ squares were cut from surface sterilized leaves and then incubated with pre-cultured agrobacteria containing p35S:oCas9-U6:gRNA-1-U6:gRNA-2 vector diluted in liquid transformation medium $(1 \times$ Murashige and Skoog basal salt mixture, $3 \%$ Sucrose, $2.0 \mathrm{mg} / \mathrm{L}$ 6-BA, $0.2 \mathrm{mg} / \mathrm{L}$ NAA, pH 5.8) with an OD at 0.6 for $10 \mathrm{~min}$ at room temperature. Then the leaf squares were laid directly onto solid transformation medium $(1 \times$ Murashige and Skoog basal salt mixture, 3\% Sucrose, $2.0 \mathrm{mg} / \mathrm{L} 6-\mathrm{BA}, 0.2 \mathrm{mg} / \mathrm{L}$ NAA, $1 \%(w / v)$ agar, $\mathrm{pH} 5.8)$ and incubated in dark. Two days later, leaf squares were transferred to selective induction medium $(1 \times$ Murashige and Skoog basal salt mixture, $3 \%$ Sucrose, $2.0 \mathrm{mg} / \mathrm{L}$ 6-BA, $0.2 \mathrm{mg} / \mathrm{L}$ NAA, $1 \%$ (w/ v) agar, $25 \mathrm{mg} / \mathrm{L}$ Hygromicin, $200 \mathrm{mg} / \mathrm{L}$ Timentin, $\mathrm{pH}$ 5.8) and incubated under light. Explants were transferred to fresh selective induction medium every 2 weeks until shoots initiated. Then the newly initiated shoots were cut from explants and transferred to root induction medium (1x Murashige and Skoog basal salt mixture, 3\% Sucrose, 1\% (w/v) agar, $25 \mathrm{mg} / \mathrm{L}$ Hygromicin, $200 \mathrm{mg} / \mathrm{L}$ Timentin, $\mathrm{pH}$ 5.8). Transgenic plants with roots were planted into soil and kept in plastic box to sustain moisture. After 2-3 weeks, transgenic plants were transferred from plastic box to growth chamber or green house. After 4 weeks, seeds were collected from transgenic plants. Cas9 transgenic $N$. benthamiana plants were as described (Yin et al. 2015).

\section{Plant growth and agroinfiltration}

Wild type and transgenic $N$. benthamiana plants were grown in growth rooms at $25^{\circ} \mathrm{C}$ under a 16-h-light/ 8 -h-dark cycle with $60 \%$ humidity.

For A. tumefaciens-mediated transient expression, GV3101 strains containing the relevant expression vectors were grown overnight at $28^{\circ} \mathrm{C}$ until $\mathrm{OD}_{600}=2.0$, then were infiltrated into $N$. benthamiana plant leaves. The infiltrated leaves from individual plants were excised at 48 to 60 hours post inoculation for the corresponding assays. For co-expression, equal amounts of A. tumefaciens cultures were mixed and used for infiltration.

For virus challenge, $\mathrm{CLCuMuV}$ or $\mathrm{CLCuMuB}$ were introduced into Agrobacterium strain GV2260. Agrobacterium cultures containing CLCuMuV or CLCuMuB were cultured and prepared as described above, then mixed at 1: 1 ratio and infiltrated into the leaves of six-leaf stage $N$. benthamiana plants.

\section{Southern blot}

CLCuMuV was detected using Southern blot. Briefly, genomic DNA was extracted from leaves of inoculated plants including control samples using a Plant Genomic DNA Extraction Kit (Tiangene, Beijing). Equal genomic DNA (10 $\mu \mathrm{g}$ DNA) of each sample was separated by $1 \%$ agarose gel with GelStain (Transgen Biotech, Beijing) at $80 \mathrm{~V}$ until the bromophenol blue visual dye had migrated approximately three-fourth of the gel. The gel was then visualized by a UV transilluminator (Proteinsimple, USA) and taken photograph, after which, the DNA in the gel was depurinated, denaturated, neutralized and transferred to a positively charged nylon membrane (Millipore Corporation, USA) by capillarity using paper tower. Then DNA was fixed on nylon membrane under UV light. Biotin probe was prepared by the random-primed labeling method using full length $\mathrm{CLCu}-$ $\mathrm{MuV}$ clone as template. Blot detection was performed using the North2South Chemiluminescent Hybridization and Detection Kit (Thermo Scientific, USA) according to the manufacturer's instructions.

\section{Confocal microscopy}

For GFP imaging, leaf discs were checked for fluorescence with a $488 \mathrm{~nm}$ laser excitation. Images were captured by using a Zeiss LSM710 inverted confocal microscope. Images were processed with Adobe Photoshop CS2.

\section{Additional files}

Additional file 1: Table S1. gRNA candidates for IR and C1 of CLCUMUV. (XLSX $12 \mathrm{~kb}$ )
Abbreviations

AZP: Artificial zinc finger protein; BGMV: Bean golden mosaic virus; BSCTV: Beet severe curly top virus; CLCUD: Cotton leaf curl disease; CLCuMuV: Cotton Leaf Curl Multan virus; CP: Capsid protein; CRISPR/ Cas: Clustered, regularly interspaced short palindromic repeats/CRISPRassociated proteins; DNA $\beta$ : Single-stranded DNA satellite; dsDNA: Doublestranded DNA; gRNA: Guide RNA; IR: Intergenic region; NHEJ: Nonhomologous end joining; PDR: Pathogen-derived resistance; RCR: Rollingcircle replication; Ren: Replication enhancer protein; Rep: Replicationassociated protein; RF: Replicative form; RGR: Ribozyme-gRNA-Ribozyme; ssDNA: Single-stranded DNA; TALE: Transcription activator like effector; TALEN: Transcription activator-like effector nucleases; TbCSV: Tobacco curly shoot virus; TLCYnV: Tomato leaf curl Yunnan virus; TrAP: Transactivator protein; TYLCCNV: Tomato yellow leaf curl China virus; TYLCSV: Tomato yellow leaf curl Sardinia virus; VIGS: Virus-induced gene silencing

\section{Acknowledgements}

We thank Prof. Caixia Gao for the helpful suggestions. As well as to Mr. Rafael A. Chavez at University of Texas at Dallas for paper revisions. 


\section{Funding}

This work was supported by the National Program on Research and Development of Transgenic Plants (2016ZX08005001, 2016ZX08009-003) and the National Natural Science Foundation of China (31700314, 31530059).

\section{Availability of data and materials}

The datasets used and analyzed during the current study are available from the corresponding author on reasonable request.

\section{Authors' contributions}

$\mathrm{TH}, \mathrm{KY}$ and $\mathrm{KX}$ performed the experiments and analyzed the data. $\mathrm{TH}$ and $\mathrm{KY}$ and $\mathrm{YL}$ designed experiments. $\mathrm{KY}$ and $\mathrm{YL}$ designed the project. $\mathrm{KY}, \mathrm{JZ}$ and $\mathrm{JS}$ wrote the manuscript. All authors have read and approved the manuscript.

\section{Ethics approval and consent to participate}

Not applicable.

\section{Consent for publication}

Not applicable

\section{Competing interests}

The authors declare that they have no competing interests.

\section{Author details}

${ }^{1}$ Center for Plant Biology, MOE Key Laboratory of Bioinformatics, School of Life Sciences, Tsinghua University, Beijing 100084, China. ${ }^{2}$ State Key Laboratory of Plant Genomics, Institute of Microbiology, Chinese Academy of Sciences, Beijing, China. ${ }^{3}$ Biology and Agriculture Research Center, University of Science and Technology Beijing, Beijing 100024, China. ${ }^{4}$ Texas A\&M AgriLife Research Center at Dallas, Texas A\&M University System, Dallas, TX 75252, USA. ${ }^{5}$ Department of Plant Pathology \& Microbiology, Texas A\&M University, College Station, TX 77843, USA.

Received: 17 December 2018 Accepted: 12 February 2019

Published online: 06 March 2019

\section{References}

Ali Z, Abulfaraj A, Idris A, Ali S, Tashkandi M, Mahfouz MM. CRISPR/Cas9-mediated viral interference in plants. Genome Biol. 2015;16:238.

Ali Z, Ali S, Tashkandi M, Zaidi SSEA, Mahfouz MM. CRISPR/Cas9-mediated immunity to geminiviruses: differential interference and evasion. Sci Rep. 2016;6:26912.

Aragão FJ, Faria JC. First transgenic geminivirus-resistant plant in the field. Nat Biotechnol. 2009:27:1086.

Aragão FJL, Ribeiro SG, Barros LMG, Brasileiro ACM, Maxwell DP, Rech EL, et al. Transgenic beans (Phaseolus vulgaris L.) engineered to express viral antisense RNAs show delayed and attenuated symptoms to bean golden mosaic geminivirus. Mol Breed. 1998:4:491-9.

Baltes NJ, Hummel AW, Konecna E, Cegan R, Bruns AN, Bisaro DM, et al. Conferring resistance to geminiviruses with the CRISPR-Cas prokaryotic immune system. Nat Plants. 2015;1:5145.

Baulcombe D. RNA silencing in plants. Nature. 2004;431:356-63.

Beachy RN. Mechanisms and applications of pathogen-derived resistance in transgenic plants. Curr Opin Biotechnol. 1997;8:215-20.

Beachy RN. Coat-protein-mediated resistance to tobacco mosaic virus: discovery mechanisms and exploitation. Philos Trans R Soc Lond Ser B Biol Sci. 1999; 354:659-64.

Briddon RW, Markham PG. Cotton leaf curl virus disease. Virus Res. 2000;71:151-9. Brustolini OJB, Machado JPB, Condori-Apfata JA, Coco D, Deguchi M, Loriato VA, et al. Sustained NIK-mediated antiviral signalling confers broad-spectrum tolerance to begomoviruses in cultivated plants. Plant Biotechnol J. 2015;13: 1300-11.

Cai JH, Xie K, Lin L, Qin BX, Chen BS, Meng JR, et al. Cotton leaf curl Multan virus newly reported to be associated with cotton leaf curl disease in China. Plant Pathol. 2010;59:794-5.

Chen W, Qian Y, Wu X, Sun Y, Wu X, Cheng X. Inhibiting replication of begomoviruses using artificial zinc finger nucleases that target viralconserved nucleotide motif. Virus Genes. 2014;48:494-501.

Cheng X, Li F, Cai J, Chen W, Zhao N, Sun Y, et al. Artificial TALE as a convenient protein platform for engineering broad-spectrum resistance to begomoviruses. Viruses. 2015;7:4772-82.
Doench JG, Fusi N, Sullender M, Hegde M, Vaimberg EW, Donovan KF, et al. Optimized sgRNA design to maximize activity and minimize off-target effects of CRISPR-Cas9. Nat Biotechnol. 2016;34:184-91.

Faria JC, Albino MM, Dias BB, Cançado LJ, da Cunha NB, Silva LDM, et al. Partial resistance to Bean golden mosaic virus in a transgenic common bean (Phaseolus vulgaris L.) line expressing a mutated rep gene. Plant Sci. 2006;171: $565-71$.

Gao Y, Zhao Y. Self-processing of ribozyme-flanked RNAs into guide RNAs in vitro and in vivo for CRISPR-mediated genome editing. J Integr Plant Biol. 2014;56: 343-9.

Hanley-Bowdoin L, Bejarano ER, Robertson D, Mansoor S. Geminiviruses: masters at redirecting and reprogramming plant processes. Nat Rev Microbiol. 2013; 11:777.

Hanley-Bowdoin L, Settlage SB, Orozco BM, Nagar S, Robertson D. Geminiviruses: models for plant DNA replication, transcription, and cell cycle regulation. Crit Rev Biochem Mol Biol. 2000;35:105-40.

Horsch RB, Fry JE, Hoffmann NL, Eichholtz D, Rogers SG, Fraley RT. A Simple and General Method for Transferring Genes into Plants. Science. 1985;277:12291231.

Hsu PD, Lander ES, Zhang F. Development and applications of CRISPR-Cas9 for genome engineering. Cell. 2014;157:1262-78.

Ji X, Zhang H, Zhang Y, Wang Y, Gao C. Establishing a CRISPR-Cas-like immune system conferring DNA virus resistance in plants. Nat plants. 2015;1:15144.

Lucioli A, Noris E, Brunetti A, Tavazza R, Ruzza V, Castillo AG, et al. Tomato yellow leaf curl Sardinia virus rep-derived resistance to homologous and heterologous geminiviruses occurs by different mechanisms and is overcome if virus-mediated transgene silencing is activated. J Virol. 2003;77: 6785-98.

Ming R, Hou S, Feng Y, Yu Q, Dionne-Laporte A, Saw JH, et al. The draft genome of the transgenic tropical fruit tree papaya (Carica papaya Linnaeus). Nature. 2008:452:991-6.

Nissim L, Perli SD, Fridkin A, Perez-Pinera P, Lu TK. Multiplexed and programmable regulation of gene networks with an integrated RNA and CRISPR/Cas toolkit in human cells. Mol Cell. 2014;54:698-710.

Ruiz MT, Voinnet O, Baulcombe DC. Initiation and maintenance of virus-induced gene silencing. Plant Cell. 1998;10:937-46.

Russell DR, Wallace KM, Bathe JH, Martinell BJ, McCabe DE. Stable transformation of Phaseolus vulgaris via electric-discharge mediated particle acceleration. Plant Cell Rep. 1993;12:165-9.

Sera T. Inhibition of virus DNA replication by artificial zinc finger proteins. J Virol. 2005;79:2614-9.

Shan Q, Wang Y, Li J, Zhang Y, Chen K, Liang Z, et al. Targeted genome modification of crop plants using a CRISPR-Cas system. Nat Biotechnol. 2013; 31:686-8.

Xie K, Minkenberg B, Yang Y. Boosting CRISPR/Cas9 multiplex editing capability with the endogenous tRNA-processing system. Proc Natl Acad Sci U S A. 2015;112:3570-5.

Yin $\mathrm{K}$, Han T, Liu G, Chen T, Wang Y, Yu AYL, et al. A geminivirus-based guide RNA delivery system for CRISPR/Cas9 mediated plant genome editing. Sci Rep. 2015:5:14926.

Zorzatto C, Machado JPB, Lopes KVG, Nascimento KJT, Pereira WA, Brustolini OJ, et al. NIK1-mediated translation suppression functions as a plant antiviral immunity mechanism. Nature. 2015;520:679-82.

Ready to submit your research? Choose BMC and benefit from

- fast, convenient online submission

- thorough peer review by experienced researchers in your field

- rapid publication on acceptance

- support for research data, including large and complex data types

- gold Open Access which fosters wider collaboration and increased citations

- maximum visibility for your research: over $100 \mathrm{M}$ website views per year

At BMC, research is always in progress.

Learn more biomedcentral.com/submission 